\title{
BMJ Open Current and possible future role of 3D modelling within oesophagogastric surgery: a scoping review protocol
}

\author{
Henry Douglas Robb (D) , ${ }^{1}$ Gemma Scrimgeour, ${ }^{2}$ Piers R Boshier, ${ }^{1}$ \\ Svetlana Balyasnikova, ${ }^{3}$ Gina Brown, ${ }^{3}$ Fernando Bello, ${ }^{1}$ \\ Christos Kontovounisios (iD) ${ }^{1,4}$
}

To cite: Robb HD,

Scrimgeour G, Boshier PR, et al. Current and possible future role of $3 \mathrm{D}$ modelling within oesophagogastric surgery: a scoping review protocol. BMJ Open 2021;11:e045546. doi:10.1136/ bmjopen-2020-045546

- Prepublication history for this paper is available online. To view these files, please visit the journal online (http://dx.doi. org/10.1136/bmjopen-2020045546).

Received 04 0ctober 2020 Accepted 12 September 2021

Check for updates

(c) Author(s) (or their employer(s)) 2021. Re-use permitted under CC BY-NC. No commercial re-use. See rights and permissions. Published by BMJ.

${ }^{1}$ Department of Surgery and Cancer, Imperial College London, London, UK

${ }^{2}$ Department of Breast Surgery, Imperial College Healthcare NHS Trust, London, UK

${ }^{3}$ Radiology Department,

Royal Marsden Hospital NHS

Foundation Trust, London, UK

${ }^{4}$ Gastrointestinal Unit, Royal

Marsden Hospital NHS

Foundation Trust, London, UK

Correspondence to

Dr Henry Douglas Robb;

henry.robb19@imperial.ac.uk

\section{ABSTRACT}

Introduction Three-dimensional (3D) reconstruction describes the generation of either virtual or physically printed anatomically accurate 3D models from twodimensional medical images. Their implementation has revolutionised medical practice. Within surgery, key applications include growing roles in operative planning and procedures, surgical education and training, as well as patient engagement and education. In comparison to other surgical specialties, oesophagogastric surgery has been slow in their adoption of this technology. Herein the authors outline a scoping review protocol that aims to analyse the current role of 3D modelling in oesophagogastric surgery and highlight any unexplored avenues for future research.

Methods and analysis The protocol was generated using internationally accepted methodological frameworks. A succinct primary question was devised, and a comprehensive search strategy was developed for key databases (MEDLINE, Embase, Elsevier Scopus and ISI Web of Science). These were searched from their inception to 1 June 2020. Reference lists will be reviewed by hand and grey literature identified using OpenGrey and Grey Literature Report. The protocol was registered to the Open Science Framework (osf.io/ta789).

Two independent reviewers will screen titles, abstracts and perform full-text reviews for study selection. There will be no methodological quality assessment to ensure a full thematic analysis is possible. A data charting tool will be created by the investigatory team. Results will be analysed to generate descriptive numerical tabular results and a thematic analysis will be performed.

Ethics and dissemination Ethical approval was not required for the collection and analysis of the published data. The scoping review report will be disseminated through a peer-reviewed publication and international conferences.

Registration details The scoping review protocol has been registered on the Open Science Framework (https:// osf.io/ta789).

\section{INTRODUCTION}

Three-dimensional (3D) reconstruction describes the generation of either virtual or physically printed anatomically accurate 3D models from two-dimensional medical images.

\section{Strengths and limitations of this study}

- This scoping review will be the first literature summarising and condensing the role of threedimensional modelling technology within oesophagogastric surgery.

- The review's thematic analysis aims to demonstrate gaps in the current literature and suggest avenues for future research by considering work done in other surgical specialties

Both the scoping review protocol and the final report will follow internationally accepted guidelines and frameworks.

- To highlight the breadth of research, rather than the depth, the review will not exclude studies based on methodological quality.

- The study will be limited to those published in English.

First described by Alberti in $1979,{ }^{1}$ the rapid technological advancement of computing and medical imaging has allowed 3D modelling to revolutionise medical practice. Within surgery, key applications include growing roles in operative planning and procedures, surgical education and training, as well as patient engagement and education. ${ }^{23}$

The benefits of 3D reconstruction within surgery are evident in a vast array of forms. From a surgical planning perspective, surgeons can now visualise complex anatomical relationships preoperatively to guide decision-making. As demonstrated by Shen et al, preoperative 3D reconstructions can improve surgical outcomes and reduce complication rates. ${ }^{4}$ Furthermore, in terms of operative procedure, the prospect of intraoperative guidance through augmented reality has become feasible through the fusion of computer-generated 3D reconstructions and the real environment. ${ }^{5}$ As the traditional apprenticeship model of surgical training becomes incompatible with modern practice and working patterns, ${ }^{6}$ physical and virtual 3D model simulations may play an increasingly 
pivotal role in surgical training. ${ }^{7}$ Since the first virtual reality (VR) simulators introduced in the $1990 \mathrm{~s},{ }^{8}$ VR simulators have grown more intricate with greater realistic haptic and stereoscopic feedback. ${ }^{3}$ Promisingly, there is a growing body of evidence that demonstrates skills developed through simulation translate to greater intraoperative surgical performance. ${ }^{9-11}$ Undoubtedly 3D modelling will become key to future surgical practice.

The advantages of 3D reconstruction are not limited to the practicing clinician and could bring a new degree of personalised medicine to the patient. It has become clear that 3D models, either virtual or 3D printed, are beneficial for patient understanding across a range of specialties. ${ }^{12-14}$ Early studies have demonstrated that patient-specific pathological 3D models could help individuals gain a greater understanding of their disease and this has the potential to improve shared decision-making. ${ }^{15}$ One can foresee a time when individualised reconstructed models will be commonplace in surgical practice, for the benefit of both patients and clinicians.

Oesophagogastric and gastrointestinal surgery, compared with subspecialties like neurosurgery and orthopaedics, have been relative 'late adopters' of 3D reconstruction techniques and technologies. ${ }^{16-18}$ There are a variety of reasons for this, including imaging-related and organ-specific complexities. ${ }^{19}$ As can be imagined, it is considerably easier to reconstruct solid bony structures in contrast to distensible and rapidly changing organs such as the stomach. However, as technologies advance and more work is done to make 3D reconstruction methodologies affordable,$^{20}$ it can be expected that $3 \mathrm{D}$ modelling will have increasing prevalence within oesophagogastric surgery.

To the authors' knowledge, there is no published literature reviewing the role of $3 \mathrm{D}$ reconstruction within oesophagogastric surgery. A scoping review is the most appropriate standardised technique to systematically analyse the use of 3D modelling in oesophagogastric surgery, identify gaps in the literature and therefore highlight avenues for future investigation. ${ }^{21}{ }^{22}$ Herein the authors outline a scoping review protocol that aims to consolidate and evaluate the current role of 3D modelling in oesophagogastric surgery, explore gaps in the literature, as well as implications for possible future practice and research.

\section{METHODS AND ANALYSIS}

The scoping review protocol was developed following the accepted six-step methodological framework created by Arksey and $\mathrm{O}^{\prime}$ Malley, ${ }^{23}$ further refined by Levac et $a l^{24}$ and the Joanna Briggs Institute (JBI) ${ }^{25}$ The Preferred Reporting Items for Systematic Reviews and MetaAnalyses-Extension for Scoping Reviews (PRISMA-ScR) checklist and guideline ${ }^{26}$ were used in the construction of this protocol and will be used in the final scoping review report.

\section{Stage 1: identify research question}

To articulate a broad yet clear primary review question, the investigatory team used the 'Population, Concept and
Context' structure as recommended the by JBI 'Manual for Evidence Synthesis'. ${ }^{27}$ This created the primary research question:

What is the current and possible future role of 3D modelling within oesophagogastric surgical practice?

By answering this question, the scoping review will be the first literature to summarise and condense the current heterogenous applications of 3D modelling within oesophagogastric surgery. As we believe oesophagogastric surgery to be a relative 'late adopter' to 3D modelling, we expect our thematic analysis to demonstrate unexplored avenues of research, in comparison to other surgical specialities. By highlighting these gaps in the literature and considering the work done in parallel specialties, we hope to recommend areas for future research and therefore possible future roles for 3D modelling within oesophagogastric surgery.

\section{Stage 2: identify studies}

A comprehensive search strategy has been developed through the collaboration of the lead investigator and a medical science librarian. After numerous pilot searches, tailored search strategies using keywords, thesauri terms (MeSH terms (MEDLINE) and EMTREE (Embase)) and Boolean operators were created for selected databases including MEDLINE, Embase, Elsevier Scopus and ISI Web of Science (see table 1). Databases are to be searched from their inception to 1 June 2020.

The reference lists from key sources identified will be hand-searched for additional studies missed through initial database searches. Grey literature will be identified through OpenGrey and Grey Literature Report.

Identified material shall be collated using EndNote V.X9 (Clarivate) prior to being transferred to Covidence, a web-based software platform for literature reviews recommended by the Cochrane Collaboration. ${ }^{28}$

\section{Stage 3: study selection}

The two-stage screening process 'title and abstract screening' and 'full-text review' will be used to identify studies for inclusion, using the Covidence platform. Two reviewers (HDR and GS) will independently screen and assess for topic relevance. Any disagreement will be resolved through discussion, and if required, a third reviewer (CK) will provide the decisive vote. Results of the selection process will be recorded on a 'Preferred Reporting Items for Systematic Reviews and Meta-Analyses (PRISMA) flow diagram'. ${ }^{29}$ Inter-rater agreement will be assessed using Cohen's kappa. ${ }^{30}$

Initially, a wide-ranging inclusion criterion will be applied (table 2); however, the study selection stage is an iterative process that may require limiting to generate a practical and applicable final study selection. This post hoc protocol development is an accepted norm in scoping reviews and any deviations will be stated in final report publication. ${ }^{31}$ For the purposes of the scoping review, oesophagogastric surgery is considered to include 


\begin{tabular}{|c|c|c|}
\hline Search & Query & Results \\
\hline 1 & Exp Oesophageal Diseases/ & 142020 \\
\hline 2 & Exp stomach diseases/ & 204194 \\
\hline 3 & Exp upper gastrointestinal tract/ & 199153 \\
\hline 4 & Exp duodenal diseases/ & 97861 \\
\hline 5 & \$oesophagus.mp. & 82392 \\
\hline 6 & Stomach/ or stomach.mp. & 239092 \\
\hline 7 & Duodenum.mp. & 58650 \\
\hline 8 & Upper gastro*.mp. & 23904 \\
\hline 9 & 1 or 2 or 3 or 4 or 5 or 6 or 7 or 8 & 537167 \\
\hline 10 & $\begin{array}{l}\text { General surgery.mp. or exp General } \\
\text { Surgery/ }\end{array}$ & 47586 \\
\hline 11 & Surgery.mp. & 2632129 \\
\hline 12 & Surgical.mp. & 1342309 \\
\hline$\underline{13}$ & Operation.mp. & 338843 \\
\hline 14 & $\begin{array}{l}\text { \$esophagectomy.mp. or exp } \\
\text { esophagectomy/ }\end{array}$ & 13749 \\
\hline 15 & Gastrectomy.mp. or exp Gastrectomy/ & 45450 \\
\hline 16 & 10 or 11 or 12 or 13 or 14 or 15 & 3181552 \\
\hline 17 & 9 and 16 & 164788 \\
\hline 18 & (3D or three dimension $\left.{ }^{\star}\right) \cdot \mathrm{mp}$. & 338859 \\
\hline 19 & $\begin{array}{l}\text { Exp imaging, three-dimensional/ or volume } \\
\text { render.mp. }\end{array}$ & 81153 \\
\hline 20 & 18 or 19 & 342110 \\
\hline 21 & 17 and 20 & 791 \\
\hline
\end{tabular}

surgery involving the stomach and oesophagus in benign and malignant states. This will also include bariatric surgery.

3D modelling is an emerging technology and it is anticipated that most research will be of low-grade evidence composed mainly of case reports and case series. Therefore, selecting studies based on methodological quality (using tools such as Newcastle-Ottawa Scale ${ }^{32}$ or ROBINS$1^{33}$ ) was deemed inappropriate as it could restrict the

\begin{tabular}{|c|c|c|}
\hline & Inclusion criteria & Exclusion criteria \\
\hline Population & $\begin{array}{l}\text { Adult patients with surgical } \\
\text { pathologies of stomach and } \\
\text { oesophagus }\end{array}$ & $\begin{array}{l}\text { Non-surgical patients } \\
\text { and pathologies, } \\
\text { paediatric patients } \\
(<16 \text { years old })\end{array}$ \\
\hline Context & $\begin{array}{l}\text { Oesophagogastric surgical } \\
\text { practice and surgical education, } \\
\text { all environments considered } \\
\text { from lab to theatre }\end{array}$ & Nil \\
\hline Concept & $\begin{array}{l}\text { All forms of 3D reconstruction, } \\
3 \mathrm{D} \text { modelling and } 3 \mathrm{D} \text { printing } \\
\text { applied to surgical practice }\end{array}$ & $\begin{array}{l}\text { 3D reconstruction } \\
\text { technologies not } \\
\text { applicable to surgical } \\
\text { practice }\end{array}$ \\
\hline Studies & $\begin{array}{l}\text { All forms of studies published in } \\
\text { peer-reviewed literature }\end{array}$ & Nil \\
\hline
\end{tabular}

3D, three-dimensional. scope of the results to a harmful degree. This is in accordance with the accepted guidance for scoping reviews. ${ }^{24}$

\section{Stage 4: charting data}

The research team will collectively develop a data charting tool. Once the data charting template has been finalised, two reviewers (HDR and GS) will independently chart data on selected studies. Any disagreement will be resolved through discussion, and if required, a third reviewer $(\mathrm{CK})$ will provide the decisive vote.

The data charting tool will follow the 'descriptiveanalytical' method described by Arksey and O'Malley. ${ }^{23}$ Fundamental study descriptors will be extracted, including

- Author(s), year of publication and study location.

- Study aims.

- Study population and sample size.

- Study design.

- Intervention.

- Main findings.

Studies will be analysed and categorised based on the reviewers' qualitative thematic summarisation of the work. The thematic summaries are expected to be divided into overarching themes found in comparative literature ${ }^{34}$ :

- Surgical education and training

- Patient education and engagement

- Surgical planning and procedure.

The data charting tool will be drafted by the research team and then piloted by the two independent reviewers (HDR and GS) over 5-10 of the selected studies for interrater agreement and relevance. Again, this is expected to be in iterative process with numerous adaptions required throughout this stage. Charting and extraction will be completed on the Covidence platform, with results transferred to Microsoft Excel (Version 365).

\section{Stage 5: collating, summarising and reporting}

As recommended by Levac et $a l,{ }^{24}$ the fifth stage will be divided into analysing data, reporting results and applying meaning to the results. Charted data from selected studies will be collated and analysed using descriptive numerical summary tables and diagrammatical mapping of the qualitative thematic analysis. A narrative descriptive summary will report the results within the context of the stated objectives and consider the implications for future practice and research.

\section{Stage 6: expert consultation}

Once results have been collated and summarised, key stakeholder experts in the field of 3D modelling shall be consulted. These consultations will provide insights beyond the current literature and provide invaluable guidance into disparities within the existing body of evidence. This will allow the research team to highlight areas for future development and advance the use of 3D modelling within oesophagogastric surgery-for the benefit of patients and clinicians alike. 


\section{Patient and public involvement}

No patient or members of the public were consulted in the generation of this protocol. However, the results may well guide the research team in their aim to improve patient engagement through 3D reconstruction.

\section{ETHICS AND DISSEMINATION}

Ethical approval was not required for the collection and analysis of the published data. The scoping review report will be disseminated through peer-reviewed publication, international and national conferences, appropriate social media channels and patient liaison groups.

Twitter Gina Brown @prof_gina_brown

Acknowledgements The authors thank Rebecca Jones, library manager and liaison librarian for the Charing Cross Campus Medical School Library at Imperial College London for her guidance and expertise in designing the draft search strategy for this scoping review.

Contributors HDR is the lead investigator and reviewer and generated the scoping review protocol. GS is a reviewer and contributed to the creation of the protocol. $\mathrm{PRB}$ and $\mathrm{SB}$ revised the draft paper and provided guidance for the scoping review. $\mathrm{GB}$ and FB provided expert consultations on the topic. CK is the senior consultant lead and assisted in the production of the protocol.

Funding The authors have not declared a specific grant for this research from any funding agency in the public, commercial or not-for-profit sectors.

Competing interests None declared.

Patient and public involvement Patients and/or the public were not involved in the design, or conduct, or reporting, or dissemination plans of this research.

Patient consent for publication Not applicable.

Provenance and peer review Not commissioned; externally peer reviewed.

Open access This is an open access article distributed in accordance with the Creative Commons Attribution Non Commercial (CC BY-NC 4.0) license, which permits others to distribute, remix, adapt, build upon this work non-commercially, and license their derivative works on different terms, provided the original work is properly cited, appropriate credit is given, any changes made indicated, and the use is non-commercial. See: http://creativecommons.org/licenses/by-nc/4.0/.

ORCID iDs

Henry Douglas Robb http://orcid.org/0000-0003-4898-2173

Christos Kontovounisios http://orcid.org/0000-0002-1828-1395

\section{REFERENCES}

1 Alberti C. Three-dimensional CT and structure models. Br J Radiol 1980;53:261-2.

2 Andolfi C, Plana A, Kania P, et al. Usefulness of three-dimensional modeling in surgical planning, resident training, and patient education. J Laparoendosc Adv Surg Tech A 2017;27:512-5.

3 Przedlacka A, Korzeniowski P, Tekkis P. 3D Simulation and Modeling for Surgeon Education and Patient Engagement. In: Atallah S, ed. Digital surgery. Cham: Springer International Publishing, 2021: 389-99.

4 Shen S, Wang P, Li X, et al. Pre-operative simulation using a threedimensional printing model for surgical treatment of old and complex tibial plateau fractures. Sci Rep 2020;10:6044.

5 Vávra P, Roman J, Zonca P, et al. Recent development of augmented reality in surgery: a review. J Healthc Eng 2017;2017:1-9.

6 Polavarapu HV, Kulaylat AN, Sun S, et al. 100 years of surgical education: the past, present, and future. Bull Am Coll Surg 2013;98:22-7.

7 Sadideen H, Hamaoui K, Saadeddin M, et al. Simulators and the simulation environment: getting the balance right in simulation-based surgical education. Int J Surg 2012;10:458-62.
8 Badash I, Burtt K, Solorzano CA, et al. Innovations in surgery simulation: a review of past, current and future techniques. Ann Trans/ Med 2016;4:453.

9 Gallagher AG, Seymour NE, Jordan-Black J-A, et al. Prospective, randomized assessment of transfer of training (TOT) and transfer effectiveness ratio (TER) of virtual reality simulation training for laparoscopic skill acquisition. Ann Surg 2013;257:1025-31.

10 Mao RQ, Lan L, Kay J, et al. Immersive virtual reality for surgical training: a systematic review. J Surg Res 2021;268:40-58.

11 Palter VN, Grantcharov TP. Individualized deliberate practice on a virtual reality simulator improves technical performance of surgical novices in the operating room: a randomized controlled trial. Ann Surg 2014;259:443-8.

12 Kim PS, Choi $\mathrm{CH}$, Han IH, et al. Obtaining informed consent using patient specific 3D printing cerebral aneurysm model. J Korean Neurosurg Soc 2019;62:398-404.

13 Zhuang Y-D, Zhou M-C, Liu S-C, et al. Effectiveness of personalized $3 \mathrm{D}$ printed models for patient education in degenerative lumbar disease. Patient Educ Couns 2019;102:1875-81.

14 Pandrangi VC, Gaston B, Appelbaum NP, et al. The application of virtual reality in patient education. Ann Vasc Surg 2019;59:184-9.

15 van de Belt TH, Nijmeijer H, Grim D, et al. Patient-Specific Actual-Size three-dimensional printed models for patient education in glioma treatment: first experiences. World Neurosurg 2018;117:e99-105.

16 Shuhaiber JH. Augmented reality in surgery. Arch Surg 2004;139:170-4.

17 Papazarkadas X, Spartalis E, Patsouras D, et al. The role of 3D printing in colorectal surgery: current evidence and future perspectives. In Vivo 2019;33:297-302.

18 Marano L, Ricci A, Savelli V, et al. From digital world to real life: a robotic approach to the esophagogastric junction with a $3 \mathrm{D}$ printed model. BMC Surg 2019;19:153.

19 Pietrabissa A, Marconi S, Negrello E, et al. An overview on 3D printing for abdominal surgery. Surg Endosc 2020;34:1-13.

20 Gómez-Ciriza G, Gómez-Cía T, Rivas-González JA, et al. Affordable three-dimensional printed heart models. Front Cardiovasc Med 2021;8:642011.

21 Sucharew H, Macaluso M. Progress notes: methods for research evidence synthesis: the scoping review approach. $J$ Hosp Med 2019;14:416-8.

22 Munn Z, Peters MDJ, Stern C, et al. Systematic review or scoping review? guidance for authors when choosing between a systematic or scoping review approach. BMC Med Res Methodol 2018;18:143.

23 Arksey H, O'Malley L. Scoping studies: towards a methodological framework. Int J Soc Res Methodol 2005;8:19-32.

24 Levac D, Colquhoun H, O'Brien KK. Scoping studies: advancing the methodology. Implement Sci 2010;5:69.

25 Peters MDJ, Godfrey CM, Khalil H, et al. Guidance for conducting systematic scoping reviews. Int J Evid Based Healthc 2015;13:141-6.

26 Tricco AC, Lillie E, Zarin W, et al. PRISMA extension for scoping reviews (PRISMA-ScR): checklist and explanation. Ann Intern Med 2018;169:467-73.

27 Peters MDJ GC, Mclnerney P, Munn Z, et al. Chapter 11: Scoping reviews: Joanna Briggs Institute, 2020. Available: https:// synthesismanual.jbi.global

28 Collaboration TC. Tools and software Cochrane community the Cochrane collaboration, 2020. Available: https://community. cochrane.org/help/tools-and-software

29 Moher D, Liberati A, Tetzlaff J, et al. Preferred reporting items for systematic reviews and meta-analyses: the PRISMA statement. BMJ 2009;339:b2535.

30 McHugh ML. Interrater reliability: the kappa statistic. Biochem Med 2012;22:276-82.

31 Lockwood C, Tricco AC. Preparing scoping reviews for publication using methodological guides and reporting standards. Nurs Health Sci 2020;22:1-4.

32 Lo CK-L, Mertz D, Loeb M. Newcastle-Ottawa scale: comparing reviewers' to authors' assessments. BMC Med Res Methodol 2014;14:45.

33 Sterne JA, Hernán MA, Reeves BC, et al. ROBINS-I: a tool for assessing risk of bias in non-randomised studies of interventions. BMJ 2016;355:i4919.

34 Wake N, Nussbaum JE, Elias MI, et al. 3D printing, augmented reality, and virtual reality for the assessment and management of kidney and prostate cancer: a systematic review. Urology 2020;143:20-32. 